\title{
Cylindrically symmetric Green's function approach for modeling the crystal growth morphology of ice
}

\author{
Kenneth G. Libbrecht* \\ Norman Bridge Laboratory of Physics, California Institute of Technology 264-33, Pasadena, California 91125
}

(Received 1 March 1999)

\begin{abstract}
We describe a front-tracking Green's function approach to modeling cylindrically symmetric crystal growth. This method is simple to implement, and with little computer power can adequately model a wide range of physical situations. We apply the method to modeling the hexagonal prism growth of ice crystals, which is governed primarily by diffusion along with anisotropic surface kinetic processes. From ice crystal growth observations in air, we derive measurements of the kinetic growth coefficients for the basal and prism faces as a function of temperature, for supersaturations near the water saturation level. These measurements are interpreted in the context of a model for the nucleation and growth of ice, in which the growth dynamics are dominated by the structure of a disordered layer on the ice surfaces. [S1063-651X(99)05708-6]

PACS number(s): 68.70.+w, 81.30.Fb, 81.10.Aj
\end{abstract}

\section{INTRODUCTION}

The dynamics of crystal growth has been well studied for the case of very fast surface kinetics, in which the growth morphology is governed primarily by diffusion and surface tension [1]. These efforts have led to a detailed "solvability theory,' in which many aspects of the growth process are well understood analytically, and there is good agreement with a wide range of experimental measurements (however, see [2]). Numerical modeling techniques have also been well developed in this regime, based both on front tracking [3-7] and phase-field modeling formalisms [8-12].

In many physical systems, however, crystal growth is dominated primarily by diffusion along with anisotropic surface kinetic processes. In this case the crystal morphology is strongly faceted, and surface tension plays a relatively minor role in the growth dynamics, serving mainly to round the edges of the facets on a small scale. The importance of anisotropic surface kinetics in environmental and industrial crystal growth is evidenced by the common occurrence of crystalline facets in these situations. Since the diffusion process is fundamentally very well understood, whereas surface kinetic processes are often not, it is desirable to disentangle the two effects. In this way measurements of crystal growth can be converted into measurements of the anisotropic surface kinetics, which can subsequently be interpreted in the context of models of nucleation and growth of the given surfaces.

An example of such a system we are considering here is ordinary ice. The crystal growth dynamics of ice has received considerable scientific attention, due in large part to its ubiquitous presence and important consequences in natural meteorological phenomena [13-17]. The growth of ice crystals from the vapor phase in particular exhibits a strongly faceted and remarkably complex morphology, with a pronounced temperature and supersaturation dependence [18].

\footnotetext{
*URL://www.its.caltech.edu/ atomic/. Electronic address: kgl@caltech.edu
}

There has been considerable speculation as to the underlying physical mechanisms responsible for this behavior, and it is widely believed that the presence of a disordered (or premelted) layer on the ice surface plays a significant role in the surface kinetics [18-20].

Our understanding of the detailed molecular dynamics of the disordered surface layer remains fairly poor, however, along with many other properties of water and ice. Recent $\mathrm{x}$-ray-diffraction observations [21] clearly indicate that the layer forms at approximately $-15^{\circ} \mathrm{C}$ on the basal faces, growing logarithmically as the temperature increases, with the layer thickness diverging at the melting temperature. The behavior on the prism faces is qualitatively similar, but with the disordering transition occurring at a somewhat higher temperature. According to the Kuroda and Lacmann (hereafter KL) model [19], the crystal growth rate depends strongly on the details of these disordered layers, since the surface structure affects (among other things) the rate of twodimensional (2D) nucleation at the smooth crystal interface. The temperature-dependent properties of the disordered layers, which are different for the prism and basal faces, are thus ultimately responsible for the complex crystal growth morphology of ice crystals. The KL model is qualitatively appealing in many of its features, but has been difficult to test quantitatively.

Although many measurements of ice crystal growth dynamics from vapor have been made under a wide range of conditions, and using different solvent gases, to date the growth data have not produced an adequate compilation of the surface kinetic coefficient as a function of temperature, supersaturation, surface orientation, and solvent gas. The reason for this is twofold. First, ice crystal growth from vapor is strongly diffusion limited, so growth velocity measurements can easily be adversely affected by experimental circumstances in which the diffusion field is not carefully controlled. Second, ice crystal growth morphologies can be quite complex, with neighboring regions of a crystal growing at very different rates. Again in such a case the structure of the diffusion field does not allow an easy interpretation of growth measurements in terms of a surface kinetic coefficient. Thus a significant first step toward a truly quantitative 
understanding of ice crystal growth, namely acquiring comprehensive measurements of the kinetic coefficient, has yet to be taken.

We outline here a potential solution to this problem involving the observation of small ice crystallites, which have a relatively simple form dominated by a solid hexagonal prism morphology $[18,22]$. In this regime the crystal growth is strongly faceted, and thus governed principally by surface kinetics and vapor diffusion. Furthermore, the 3D diffusion field is nearly cylindrically symmetric and can be adequately modeled using the Green's function approach described below. Using such a modeling procedure we demonstrate that the surface kinetic coefficients can be derived with considerable accuracy from ice crystal growth data.

Numerical modeling of solidification has undergone considerable development work in the past few years, both for front-tracking [3-7] and phase-field methods [8-12]. The computational difficultly of the solidification problem is considerable, however, and full 3D calculations have only recently been performed using efficient adaptive-grid phasefield techniques $[9,12]$. The $3 \mathrm{D}$ calculations require substantial computer power, and have not been demonstrated for low supersaturation levels or in the case of strongly anisotropic surface kinetics. Fully mathematically consistent modeling of strongly faceted surfaces has been a particularly challenging problem in crystal growth [24,25].

Green's function techniques have been well developed in two dimensions, both for surface-tension-limited growth $[3,23]$ and for surface-kinetic-limited growth [4]. Here we extend these treatments to the quasi-2D case of cylindrically symmetric growth, which mainly involves the choice of a different Green's function, along with some special treatment near the axis of the cylindrical coordinates. The approach described here is quite simple to implement, requires little computer power, and can be adapted to a wide range of physical situations. Furthermore, it is a fairly good approximation to hexagonal prism growth, as long as there is not a substantial hollowing of the growth of the prism faces. Thus this approach applies fairly well to the case of ice crystal growth when the supersaturation is near or below the water saturation value, or when the crystal size is not too large. It is also well suited to model the hollow column morphology [18], in which there is typically a substantial, nearly cylindrically symmetric, hollowing of the basal faces, but little hollowing of the prism faces.

We have used this technique here to model the growth of small ice crystallites grown in air, using data from Yamashita $[18,22]$. We derive from these data, for a supersaturation near the water saturation level, the kinetic growth coefficient as a function of temperature, which exhibits a great deal of structure between $0{ }^{\circ} \mathrm{C}$ and $-30^{\circ} \mathrm{C}$. We interpret the kinetic coefficient measurements in the context of growth models in the presence of a disordered layer on the different crystal facets of ice. Additional data of this kind, at different supersaturation levels and in different solvent gases, should reveal much about the underlying physics governing the growth of ice crystals, and may additionally contribute to our understanding of the enigmatic surface structure of ice.

\section{CYLINDRICALLY SYMMETRIC CRYSTAL GROWTH}

\section{A. The diffusion equation and boundary conditions}

For concreteness we define our problem to be crystal growth from a supersaturated vapor in a solvent gas, al- though the notation could easily be generalized to other systems [26]. Vapor transport through the solvent gas is governed by the diffusion equation

$$
\frac{\partial c}{\partial t}=D \nabla^{2} c
$$

where $D$ is the diffusion constant and $c$ is some generalized concentration, which we take here to be number density of solute molecules. Since the solid density is much higher than the vapor density $\left(c_{\text {solid }} \gg c_{\text {vapor }}\right)$, the diffusion length $l_{D}$ $\equiv 2 \mathrm{D} / \mathrm{v}$ is typically very large, which allows us to ignore the time derivative in the above, leaving $\nabla^{2} c=0$ outside the crystal surface.

Our first boundary condition for the solution of this equation arises far away from the growing crystal, where we set $c(\infty)=c_{\infty}$, the supersaturation level. Another boundary condition comes from mass conservation, which gives

$$
\mathrm{v}_{n} \equiv\left(\hat{n} \cdot \overrightarrow{\mathrm{v}}_{\text {surf }}\right)=\frac{D}{c_{\text {solid }}}(\hat{n} \cdot \vec{\nabla} c)_{\text {surf }}
$$

at the crystal surface, where $\hat{n}$ is the normal to the surface, $\vec{v}_{\text {surf }}$ is the surface growth velocity vector, and the expression on the right-hand side is evaluated just above the crystal surface. Another expression for the growth velocity comes from the Wilson-Frankel law,

$$
\mathrm{v}_{n}=K \frac{c_{\mathrm{surf}}-c_{\mathrm{eq}}}{c_{\mathrm{eq}}}
$$

where $c_{\text {surf }}$ is the number density just above the surface, $c_{\text {eq }}$ is the number density above an equilibrium surface (for which $\mathrm{v}_{n}=0$ ), and $K$ is the usual kinetic coefficient. The maximum value of the kinetic coefficient comes from the Hertz-Knudsen formula,

$$
K_{\max }^{\prime}=\alpha K_{\max }=\frac{\alpha P_{\mathrm{eq}} V_{m}}{(2 \pi m k T)^{1 / 2}}
$$

where $\alpha \leqslant 1$ is the sticking coefficient, $P_{\text {eq }}$ is the vapor pressure over an equilibrium surface, $V_{m}$ is the molecular volume, and $m$ is the molecular mass.

Since we are interested mainly in growth limited by diffusion and surface kinetics, we ignore surface tension for the present by taking $c_{\text {eq }}=c_{\text {sat }}$, where $c_{\text {sat }}$ is the saturation vapor pressure above a flat surface $[4,23]$. Then defining $\sigma \equiv(c$ $\left.-c_{\text {sat }}\right) / c_{\text {sat }}$, the diffusion equation becomes

$$
\nabla^{2} \sigma=0
$$

with the boundary conditions $\sigma(\infty)=\sigma_{\infty}$ and $\mathrm{\vee}_{n}=K \sigma_{\text {surf }}$. Combining the two expressions for $\mathrm{v}_{n}$ yields the mixed boundary condition

$$
K \sigma_{\text {surf }}=\frac{c_{\text {sat }}}{c_{\text {solid }}} D(\hat{n} \cdot \vec{\nabla} \sigma)_{\text {surf }} .
$$

For the present case we consider that $K=K\left(\hat{\theta}, T, \sigma_{\text {surf }}\right)$ can depend on crystallographic orientation $\hat{\theta}$ of the growing surface, temperature $T$, and the supersaturation value $\sigma_{\text {surf }}$.

For the functional form of the kinetic coefficient, we take 


$$
K=K_{\max } \eta_{1} \tanh \left(\frac{1}{\eta_{1}}\right) \eta_{2} \tanh \left(\frac{1}{\eta_{2}}\right)
$$

with

$$
\eta_{i}=\left(\tan \theta_{i}+s_{i} \epsilon_{i}\right) / s_{i},
$$

where $\theta_{i}$ is the angle of the surface normal relative to the crystal axis, and the subscripts refers to either the $\hat{z}$ (basal) or $\hat{r}$ (prism) axes. This form is similar to that used by Yokoyama and Kuroda [4], which derives from the advancement of admolecule terraces calculated by Burton, Cabrera, and Frank [29]. For small $s_{i}$ this form provides $K \approx K_{\max }$ except near $\theta_{i}=0$, where there is a cusplike behavior. Here we have softened the cusp by adding the $\epsilon_{i}$ factor, which gives $K \approx \epsilon_{i} K_{\max }$ on a facet.

\section{B. Green's function formalism}

The complexity of diffusion limited growth, even for the cylindrically symmetric case, is such that an analytic solution is nearly impossible for anything but the very simplest cases, requiring the use of numerical simulation. We use a Green's function method here, which is an extension of that described for the $2 \mathrm{D}$ case $[3,4,23]$. With this technique we transform the bulk diffusion equation into an integrodifferential equation over the crystal interface. By solving this interface equation numerically, the grid points move with the interface, and the transformation reduces the dimensionality of the problem, in our case from a $2 \mathrm{D}$ to a $1 \mathrm{D}$ problem.

The Green's function formalism for transforming the Laplace equation is well known from electrostatics [27], and we apply it here to the cylindrically symmetric problem. From Green's theorem, using Jackson's notation in 3D,

$$
\int_{V}\left(\phi \nabla^{2} \psi-\psi \nabla^{2} \phi\right) d V=\oint_{S}\left[\phi \frac{\partial \psi}{\partial n}-\psi \frac{\partial \phi}{\partial n}\right] d A,
$$

where $\phi$ and $\psi$ are two scalar functions, and the volume and area integrals are, respectively, over some volume $V$ and the surface of that volume $S$. The derivatives are with respect to the surface normal, i.e., $(\partial \phi / \partial n)=(\hat{n} \cdot \vec{\nabla} \phi)$. Taking our lead from electrostatics, we take $\phi=\sigma$, and let our volume be the space between the crystal surface and the far-away boundary. The Green's function for the cylindrically symmetric case is

$$
\psi\left(\vec{x}, \vec{x}^{\prime}\right)=\frac{\epsilon_{0}}{Q} \Phi_{\text {ring }}\left(\vec{x}, \vec{x}^{\prime}\right)
$$

where $\Phi_{\text {ring }}\left(\vec{x}, \vec{x}^{\prime}\right)$ is the electrostatic potential at $\vec{x}$ arising from a ring of charge $Q$ at position $\vec{x}^{\prime}$. Here our variables are $\vec{x}=(r, z)$ in cylindrical coordinates, and likewise for $\vec{x}^{\prime}$.

Taking $\vec{x}$ to be just above the crystal surface, and using $\nabla^{2} \sigma=0, \nabla^{2} \psi=-\delta\left(\vec{x}-\vec{x}^{\prime}\right)$, and our additional boundary conditions, we obtain

$$
\begin{aligned}
-\sigma(\vec{x})= & \oint_{S}\left[\sigma \frac{\partial \psi}{\partial n^{\prime}}-\psi \frac{\partial \sigma}{\partial n^{\prime}}\right] d A^{\prime}=\int\left[\sigma \frac{\partial \psi}{\partial n^{\prime}}-\psi \frac{\partial \sigma}{\partial n^{\prime}}\right] d A_{1}^{\prime} \\
& +\int\left[\frac{\partial \psi}{\partial n^{\prime}}-\psi \frac{c_{\text {solid }}}{D c_{\text {sat }}} K(\hat{\theta}, \sigma)\right] \sigma d A_{2}^{\prime},
\end{aligned}
$$

where $A_{1}^{\prime}$ is the boundary at infinity and $A_{2}^{\prime}$ is the boundary at the crystal surface.

To evaluate the integrals at infinity, we note the spherically symmetric solution for a crystal of radius $R_{0}$,

$$
\sigma(R)=\sigma_{\infty}-\frac{\Delta \sigma R_{0}}{R}
$$

where here $R$ is the radius in spherical coordinates. Thus for large $R$ we have $\sigma \rightarrow \sigma_{\infty}$ and $d \sigma / d n \sim R^{-2}$. Similarly, $\psi$ $\sim R^{-1}$ and $d \psi / d n=d \psi / d r \sim R^{-2}$. The area of the boundary goes like $A_{1} \sim R^{2}$, so the second term in the first square brackets above vanishes and the first term becomes

$$
\int\left[\sigma \frac{\partial \psi}{\partial n^{\prime}}\right] d A_{1}^{\prime} \rightarrow-\sigma_{\infty}
$$

and so

$$
\begin{aligned}
\sigma(\vec{x}) & =\sigma_{\infty}-\int\left[\frac{\partial \psi}{\partial n^{\prime}}-\psi \frac{c_{\text {solid }}}{D c_{\text {sat }}} K(\hat{\theta}, \sigma)\right] \sigma d A_{2}^{\prime} \\
& =\sigma_{\infty}+2 \pi \int\left[\frac{\partial \psi}{\partial n^{\prime}}-\psi \frac{c_{\text {solid }}}{D c_{\text {sat }}} K(\hat{\theta}, \sigma)\right] \sigma r^{\prime} d \Gamma_{2}^{\prime},
\end{aligned}
$$

where the latter integral is a line integral over the boundary of the cylindrical crystal. The sign change in this term comes from an arbitrary convention as to the direction of the line integral over $\Gamma_{2}$. We note this form is somewhat different from that described in Ref. [4]. In the case of very fast kinetics, the boundary conditions change, giving

$$
\sigma(\vec{x})=\sigma_{\infty}+2 \pi \int\left[\frac{\partial \psi}{\partial n^{\prime}} \sigma-\psi \frac{\partial \sigma}{\partial n^{\prime}}\right] r^{\prime} d \Gamma_{2}^{\prime},
$$

where again we have that $\vec{x}$ lies just above the crystal surface.

\section{The Green's function}

Since there is no simple analytic expression for $\Phi_{\text {ring }}\left(\vec{x}, \vec{x}^{\prime}\right)$, we resort to numerical techniques to evaluate it and its derivatives. For this we start with a ring of charge $Q$ and radius $r^{\prime}=r_{0}$, located at $z^{\prime}=0$,

$$
\Phi_{0}(r, z)=\Phi_{\text {ring }}\left((r, z),\left(r_{0}, 0\right)\right),
$$

which we generate in table form using a commercial adaptive grid electrostatics program [28] using $Q=10^{-9} \mathrm{C}$ and $r_{0}=1 \mathrm{~m}$. Translation in $z$ gives

$$
\begin{aligned}
\Phi_{\text {ring }}\left((r, z),\left(r_{0}, z^{\prime}\right)\right) & =\Phi_{\text {ring }}\left(\left(r, z-z^{\prime}\right),\left(r_{0}, 0\right)\right) \\
& =\Phi_{\text {ring }}\left(\left(r,\left|z-z^{\prime}\right|\right),\left(r_{0}, 0\right)\right)
\end{aligned}
$$


where we have used the fact that the ring potential is symmetric in $z$ about the ring plane. Similarly, scaling in $r^{\prime}$ yields

$$
\Phi_{\text {ring }}\left((r, z),\left(r^{\prime}, 0\right)\right)=\left(\frac{r_{0}}{r^{\prime}}\right) \Phi_{\text {ring }}\left(\left(r \frac{r_{0}}{r^{\prime}}, z \frac{r_{0}}{r^{\prime}}\right),\left(r_{0}, 0\right)\right)
$$

so for an arbitrary ring with charge $Q$,

$$
\begin{aligned}
\Phi_{\text {ring }}\left((r, z),\left(r^{\prime}, z^{\prime}\right)\right) & =\left(\frac{r_{0}}{r^{\prime}}\right) \Phi_{\text {ring }}\left(\left(r \frac{r_{0}}{r^{\prime}},\left|z-z^{\prime}\right| \frac{r_{0}}{r^{\prime}}\right),\left(r_{0}, 0\right)\right) \\
& =\left(\frac{r_{0}}{r^{\prime}}\right) \Phi_{0}\left(r \frac{r_{0}}{r^{\prime}},\left|z-z^{\prime}\right| \frac{r_{0}}{r}\right)
\end{aligned}
$$

and the Green's function becomes

$$
\psi\left(\vec{x}, \vec{x}^{\prime}\right)=\frac{\epsilon_{0}}{Q}\left(\frac{r_{0}}{r^{\prime}}\right) \Phi_{0}\left(r \frac{r_{0}}{r^{\prime}},\left|z-z^{\prime}\right| \frac{r_{0}}{r^{\prime}}\right) .
$$

Near the ring itself the potential looks like that of a line charge, so

$$
\Phi_{0} \rightarrow \frac{-Q}{4 \pi^{2} r_{0} \epsilon_{0}} \ln \left(\frac{\gamma R}{r_{0}}\right) \approx-\left(\begin{array}{ll}
2.863 & \mathrm{~V}
\end{array}\right) \ln \left(\frac{R}{8 r_{0}}\right)
$$

using the above values for $Q$ and $r_{0}$. Here we have defined a local Cartesian coordinate system $(\rho, \zeta)=\left(r-r_{0}, z-z_{0}\right)$, and define the length variable $R=\left(\rho^{2}+\zeta^{2}\right)^{1 / 2}$.

Since the ring is curved, we can do a bit better at matching the numerical function by adding a linear term in $r$. Thus we take

$$
\Phi_{0}(r, z) \rightarrow-\left(A_{2}-A_{1} \rho\right) \ln \left(\frac{R}{8 r_{0}}\right),
$$

where $A_{1}=1.0822, A_{2}=2.863$, and $\rho$ is in meters. The value of $A_{1}$ was determined by a fit to the numerical data. The gradient of $\Phi_{0}$ near the ring is then $\nabla \Phi_{0}$ $=\left(\partial \Phi_{0} / \partial r, \partial \Phi_{0} / \partial z\right)$, with

$$
\frac{\partial \Phi_{0}}{\partial r}=-\left(A_{2}-A_{1} \rho\right) \frac{\rho}{R^{2}}+A_{1} \ln \left(\frac{R}{8 r_{0}}\right)
$$

and

$$
\frac{\partial \Phi_{0}}{\partial z}=-\left(A_{2}-A_{1} \rho\right) \frac{\zeta}{R^{2}} .
$$

We use this form for $\Phi_{0}$ when $R<0.06 \mathrm{~m}$.

The asymptotic behavior of the ring potential at large distance can be gotten from a simple multipole expansion, keeping only the monopole and quadrupole $(l, m)=(2,0)$ terms. To this order

$$
\Phi_{0}(r, z) \approx \frac{Q}{4 \pi \epsilon_{0} \Omega}\left[1-\frac{1}{4} \frac{r_{0}^{2}}{\Omega^{2}}\left(3 \cos ^{2} \theta-1\right)\right]
$$

and this form is used whenever $\Omega=\left(r^{2}+z^{2}\right)^{1 / 2}>4 \mathrm{~m}$. We have evaluated $\Phi_{0}$, comparing the tabulated and asymptotic forms, and estimate that with the above procedure we compute the Green's function everywhere to an accuracy of

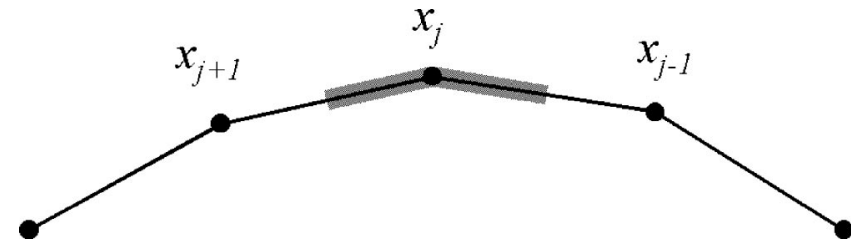

FIG. 1. A section of the polygonal crystal surface. The shaded area denotes the integration region for point $x_{j}$.

roughly a few parts in $10^{3}$. We also verify that our Green's function satisfies the identity $\psi\left(\vec{x}, \vec{x}^{\prime}\right)=\psi\left(\vec{x}^{\prime}, \vec{x}\right)$ to the same accuracy.

To compute the gradient of $\psi\left(\vec{x}, \vec{x}^{\prime}\right)$, we can use the fact that $\nabla_{x^{\prime}} \psi\left(\vec{x}, \vec{x}^{\prime}\right)=\nabla_{x^{\prime}} \psi\left(\vec{x}^{\prime}, \vec{x}\right)$ to write

$$
\frac{\partial}{\partial r^{\prime}} \psi\left(\vec{x}, \vec{x}^{\prime}\right)=\frac{\epsilon_{0}}{Q}\left(\frac{r_{0}}{r}\right)^{2} \nabla_{x 1} \Phi_{0}\left(r^{\prime} \frac{r_{0}}{r},\left|z-z^{\prime}\right| \frac{r_{0}}{r}\right),
$$

where $\nabla_{x 1} \Phi_{0}\left(x_{1}, x_{2}\right)$ is the derivative of $\Phi_{0}$ with respect to its first argument. Similarly

$$
\begin{aligned}
\frac{\partial}{\partial z^{\prime}} \psi\left(\vec{x}, \vec{x}^{\prime}\right)= & \frac{\epsilon_{0}}{Q}\left(\frac{r_{0}}{r}\right)^{2} \nabla_{x 2} \Phi_{0}\left(r^{\prime} \frac{r_{0}}{r},\left|z-z^{\prime}\right| \frac{r_{0}}{r}\right) \\
& \times \operatorname{sgn}\left(z^{\prime}-z\right) .
\end{aligned}
$$

\section{NUMERICAL SIMULATION}

Given the above Green's function formalism for solving the cylindrically symmetric crystal growth problem, we now write down a numerical algorithm. The basic steps are as follows [3,23]:

(i) Pick an initial crystal shape, with a discrete set of $N$ points $\vec{x}_{i}$.

(ii) Calculate the various pieces of Eq. (5), and turn this integral equation into a matrix equation $\sigma_{i}=\sigma_{0}+H_{i j} \sigma_{j}$, where $\sigma_{i}$ is the vector of supersaturation values on the surface points $x_{i}$.

(iii) Solve the matrix equation for $\sigma_{i}$ and calculate the crystal growth velocity vector $\mathrm{v}_{i}$.

(iv) Evolve the crystal profile points $x_{i}$ by a distance $\mathrm{v}_{i} \Delta t$ along the surface normal.

(v) Change the spacing and distribution of $x_{i}$ along the surface, if desired.

(vi) Iterate.

\section{A. Defining the matrix equation}

We break up the integral equation above [Eq. (5)] into a sum $[3,23]$ :

$$
\begin{aligned}
\sigma\left(\vec{x}_{i}\right)= & \sigma_{\infty}+\sum_{j=1}^{N} \int_{\left(x_{j}+x_{j-1}\right) / 2}^{\left(x_{j}+x_{j+1}\right) / 2}\left[\frac{\partial \psi}{\partial n^{\prime}}-\psi \frac{c_{\text {solid }}}{D c_{\text {sat }}} K(\hat{\theta}, \sigma)\right] \\
& \times 2 \pi r^{\prime} \sigma d \Gamma_{2}^{\prime},
\end{aligned}
$$

where the $x_{j}$ in the integral limits are meant as the vector points shown in Fig. 1. This can then be written

$$
\sigma_{i}=\sigma_{\infty}+\sum_{j=1}^{N} H_{i j} \sigma_{j}
$$


where

$$
\begin{aligned}
H_{i j}= & \int_{\left(x_{j}+x_{j-1}\right) / 2}^{\left(x_{j}+x_{j+1}\right) / 2}\left[\frac{\partial \psi}{\partial n^{\prime}}\left(x_{i}, x^{\prime}\right)\right. \\
& \left.-\psi\left(x_{i}, x^{\prime}\right) \frac{c_{\text {solid }}}{D c_{\text {sat }}} K(\hat{\theta}, \sigma)\right] 2 \pi r^{\prime} d \Gamma_{2}^{\prime} \\
= & A_{i j}-B_{i j} \\
= & \left(A_{i j}^{\prime}+A_{i j}^{\prime \prime}\right)-\left(B_{i j}^{\prime}+B_{i j}^{\prime \prime}\right)
\end{aligned}
$$

and

$$
\begin{gathered}
A_{i j}^{\prime}=\int_{\left(x_{j}+x_{j-1}\right) / 2}^{x_{j}} \frac{\partial \psi}{\partial n^{\prime}}\left(x_{i}, x^{\prime}\right) 2 \pi r^{\prime} d \Gamma_{2}^{\prime} \\
B_{i j}^{\prime}=\frac{c_{\text {solid }}}{D c_{\text {sat }}} \int_{\left(x_{j}+x_{j-1}\right) / 2}^{x_{j}} K\left(\hat{\theta}_{j-1}, \sigma_{j}\right) \psi\left(x_{i}, x^{\prime}\right) 2 \pi r^{\prime} d \Gamma_{2}^{\prime} \\
A_{i j}^{\prime \prime}=\int_{x_{j}}^{\left(x_{j}+x_{j+1}\right) / 2} \frac{\partial \psi}{\partial n^{\prime}}\left(x_{i}, x^{\prime}\right) 2 \pi r^{\prime} d \Gamma_{2}^{\prime} \\
B_{i j}^{\prime \prime}=\frac{c_{\text {solid }}}{D c_{\text {sat }}} \int_{x_{j}}^{\left(x_{j}+x_{j+1}\right) / 2} K\left(\hat{\theta}_{j}, \sigma_{j}\right) \psi\left(x_{i}, x^{\prime}\right) 2 \pi r^{\prime} d \Gamma_{2}^{\prime}
\end{gathered}
$$

with

$$
\theta_{j}=\arctan \left(\frac{r_{j+1}-r_{j}}{z_{j+1}-z_{j}}\right)
$$

Fundamentally this is a nonlinear equation, owing to the dependence of $K$ on $\sigma$. However, we assume that $K$ depends only weakly on $\sigma$, so we can use the previous value of $\sigma_{i}$ in computing $K$.

We evaluate these integrals using Gaussian quadratures, using the usual form

$$
\int_{-1}^{+1} f(\xi) d \xi \approx \sum_{m=1}^{n} w_{m} f\left(\xi_{m}\right)
$$

For example, we can rewrite one of the terms above in the form

$$
B_{i j}^{\prime \prime} \approx \frac{2 \pi c_{\text {solid }}}{D c_{\text {sat }}} \frac{s_{j}}{4} K\left(\hat{\theta}_{j}, \sigma_{j}\right) \int_{-1}^{+1} \psi\left(x_{i}, x^{\prime}(\xi)\right) r^{\prime}(\xi) d \xi
$$

where $s_{j}=\left|x_{j+1}-x_{j}\right|$ and

$$
x^{\prime}(\xi)=\left(\frac{3-\xi}{4}\right) x_{j}+\left(\frac{1+\xi}{4}\right) x_{j+1}
$$

and we obtain similar forms for the other terms. For evaluating $B_{i j}^{\prime \prime}$, which has a logarithmic divergence in the integrand, we use the logarithmic Gaussian quadrature formula $[3,23]$. The end points also present some special cases since $r_{i} \rightarrow 0$, and must be done separately. For example, we have

$$
B_{i 1} \approx \frac{c_{\text {solid }}}{D c_{\text {sat }}} K\left(\frac{\pi}{2}, \sigma_{1}\right) \psi\left(x_{i}, x_{1}\right) \pi\left(\frac{r_{2}}{2}\right)^{2} .
$$

We evaluate the normal derivatives similarly, giving, for example, to lowest order

$$
\begin{aligned}
A_{i j}^{\prime \prime} \approx & \pi \frac{\epsilon_{0}}{Q}\left(\frac{r_{0}}{r_{i}}\right)^{2}\left[\nabla_{x 1} \Phi_{0}\left(r^{\prime} \frac{r_{0}}{r_{i}},\left|z_{i}-z^{\prime}\right| \frac{r_{0}}{r_{i}}\right)\left(-z_{j+1}+z_{j}\right)\right. \\
& +\nabla_{x 2} \Phi_{0}\left(r^{\prime} \frac{r_{0}}{r_{i}},\left|z_{i}-z^{\prime}\right| \frac{r_{0}}{r_{i}}\right) \\
& \left.\times \operatorname{sgn}\left(z^{\prime}-z_{i}\right)\left(r_{j+1}-r_{j}\right)\right] r^{\prime}
\end{aligned}
$$

where

$$
x^{\prime}=\frac{3}{4} x_{j}+\frac{1}{4} x_{j+1}
$$

and for the end points,

$$
A_{i 1} \approx \frac{\epsilon_{0}}{Q}\left(\frac{r_{0}}{r_{i}}\right)^{2} \nabla_{x 2} \Phi_{0}\left(0,\left|z_{i}-z_{1}\right| \frac{r_{0}}{r_{i}}\right) \operatorname{sgn}\left(z_{1}-z_{i}\right) \pi\left(\frac{r_{2}}{2}\right)^{2}
$$

Finally, for the diagonal elements we use the sum rule [23] to write

$$
A_{i i}=-\sum_{j \neq i} A_{i j}
$$

Implementation of the above into computer code was straightforward, but some care had to be taken to avoid instabilities. The first of these is intrinsic to the problem, since we have ignored surface tension, which is known to be the dominant effect limiting the Mullens-Sekerka instability in many crystal growth problems [23]. This instability does not have a detrimental effect in the case of a highly anisotropic kinetic coefficient, however since then the crystal growth is strongly faceted [4]. The numerical procedure described above does not introduce additional instabilities at the corners of the facets.

Another problem arises when the $s_{i}$ are very small, and the kinetic coefficient is thus highly anisotropic. In this circumstance the growth of a polygonal line segment on the crystal surface can take it from $\theta_{i}<0$ to $\theta_{i}>0$ (or vice versa) in a single time step, not stopping at $\theta_{i}=0$, where $K$ assumes its minimum value. Taking very small time steps solves this problem, but at a price of greatly increased execution time for a given run. We eliminated this problem even for large time steps by checking at each iteration if any line segment is going to advance and changes the sign of $\theta_{i}$ in the process. If so, the growth of one of the vertex points is reduced to force $\theta_{i} \rightarrow 0$ for that segment. This ensures that the surface does not pass through the "magic angles" which define the crystal facets without pausing at $\theta_{i}=0$ for at least one time step.

Finally, we find some indication of numerical instabilities when a large number of interface points is used, typically greater than a few hundred. We believe these problems arise because of the large matrix inversion that results [3], along with our imperfect computation of the ring charge Green's function described above. With these caveats, we have found that the Green's function method yields quite robust and reproducible results for the growth of prismatic crystals. 


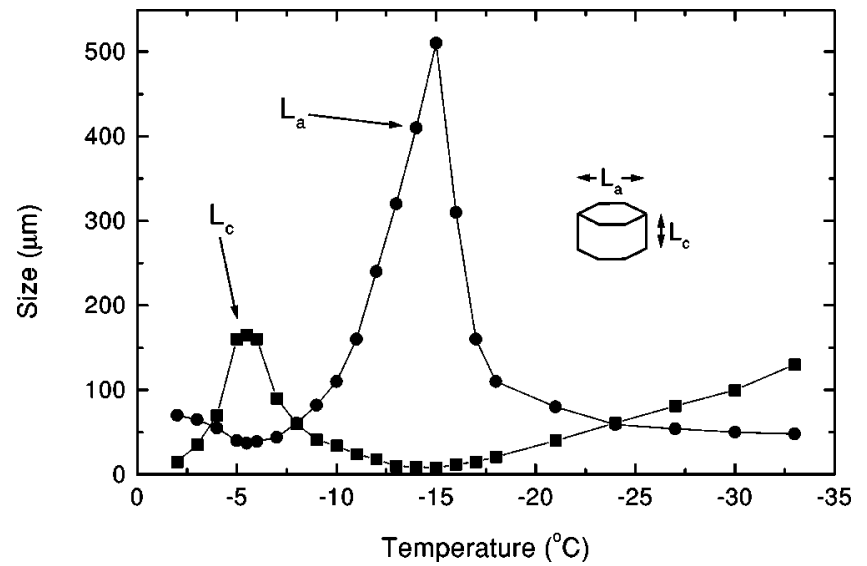

FIG. 2. Ice crystal growth data, adapted from Yamashita $[18,22]$. This shows the ice prism diameter $\left(L_{a}\right)$ and thickness $\left(L_{c}\right)$ after growing $200 \mathrm{sec}$ in ordinary air supersaturated at the water saturation level.

\section{APPLICATION TO THE GROWTH MORPHOLOGY OF ICE}

Diameter measurements of growing ice crystallites are shown in Fig. 2, which is adapted from the data of Yamashita $[18,22]$. These measurements were obtained by seeding ice crystals in a variable-temperature cloud chamber operating with ordinary air, saturated with suspended water droplets. The presence of water droplets, similar to those in a natural cloud, guaranteed that the water vapor pressure in the chamber was fixed very near the water saturation level. After nucleating a large collection of crystallites, the suspended crystallites were allowed to grow for $200 \mathrm{sec}$, at which point a sample of the crystal population was collected and the crystal sizes measured. These data clearly show the well-known morphology transitions from platelike growth $\left(-2^{\circ} \mathrm{C}\right)$ to columnar growth $\left(-5^{\circ} \mathrm{C}\right)$ to platelike growth $\left(-15^{\circ} \mathrm{C}\right)$ and back to columnar growth $\left(-30^{\circ} \mathrm{C}\right)$. These data are particularly well suited for quantitative analysis, since the crystals grew in the absence of perturbing boundary conditions, in contrast to crystal growth on substrates or other fixtures.

To model these data, we took $s_{1}=s_{2}=1 / 100, D=2$ $\times 10^{-5} \mathrm{~m}^{2} / \mathrm{sec}^{-1}$, and set the saturation at infinity equal to the water saturation level. Because the crystal growth was typically strongly faceted, the precise values of $s_{1}$ and $s_{2}$ were not critical for determining the facet growth rate. The initial shape of the crystal was spherical with a radius of five $\mu \mathrm{m}$, which was also not critical. Then $\epsilon_{1}$ and $\epsilon_{2}$ in Eq. (3) were adjusted so that the crystal dimensions at the end of 200 sec of growth most closely matched the measured values, assuming that the kinetic coefficient was independent of $\sigma$ for these calculations. From this analysis we derived values for the kinetic coefficient on the prism and basal faces as a function of temperature, for supersaturation values of order the water saturation level, as shown in Fig. 3. By running models with different input parameters, we estimate the intrinsic modeling uncertainties to be roughly $20 \%$ for most of the data.

The modeled crystal growth showed some hollowing of the basal faces, but mainly exhibited simple prismatic growth; the crystal size was taken to equal the largest dimension in all cases, i.e., $L_{a}=2 r_{\max }$ and $L_{c}=\left(z_{\max }-z_{\min }\right)$. We

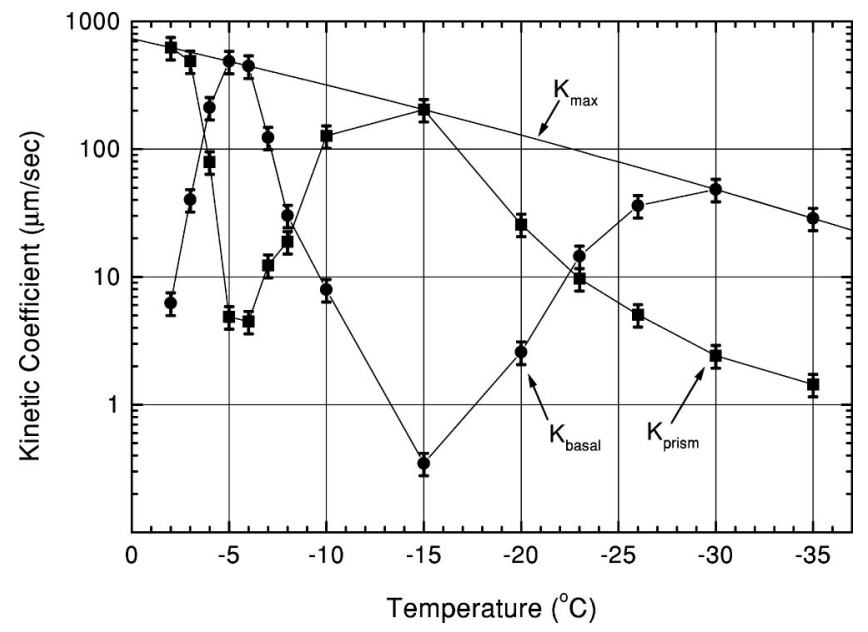

FIG. 3. Kinetic coefficient measurements derived from the data in Fig. 2, obtained by numerical modeling of cylindrically symmetric growth, as described in the text. The line for $K_{\max }$ shows the maximum kinetic coefficient allowed by the Hertz-Knudsen formula. The strong temperature dependence in $K_{\text {basal }}$ and $K_{\text {prism }}$ arises from the unusual surface properties of ice, which are ultimately responsible for the complex growth morphology of ice crystals.

were able to reproduce the observed crystal sizes everywhere except at $-15^{\circ} \mathrm{C}$, where the calculated values of $L_{a}$ after $200 \mathrm{sec}$ were too small even with $K_{\text {prism }}=K_{\max }$. It is likely that some dendritelike growth (hollowing of the prism facets) occurred at this temperature, thus producing experimentally measured crystal diameters in excess of what one would produce using a cylindrically symmetric model. The observed growth may also have been affected by the ventilation effect [30], since the crystallites were tumbling in the cloud chamber during the growth phase. Thus for $T=-15^{\circ} \mathrm{C}$ only we took $K_{\text {prism }}=K_{\text {max }}$ and $K_{\text {basal }} \approx L_{c} / \tau \sigma_{\infty}$ in Fig. 3, where $\tau=200 \mathrm{sec}$ is the growth time. At all other temperatures shown in Fig. 3, we could reproduce the observed $L_{a}$ and $L_{c}$, and the fact that $K$ was bounded by $K_{\max }$ arose naturally out of the model.

These effects introduce some systematic uncertainty into the inferred kinetic coefficients in Fig. 3, so the results here must be taken as preliminary. Additional data are needed, particularly under very controlled conditions at different supersaturation levels, as might be obtained from observations of levitated growing crystals [31]. With such data one could observe the crystal size continuously during growth, and compare the resultant crystal morphology with the model calculations as a function of time.

The results in Fig. 3 can be compared with the KL model predictions for growth in the presence of a disordered surface layer [19]. For example, one feature of the KL model is that for $T \lesssim-15^{\circ} \mathrm{C}$, the disordered layer is absent on both the basal and prism facets, and the growth kinetics is governed by the $2 \mathrm{D}$ nucleation rate. In particular, the onset of rapid $2 \mathrm{D}$ nucleation is thought to produce the sharp increase in $K_{\text {basal }}$ for $T<-15^{\circ} \mathrm{C}$, which rises to $K_{\text {basal }}=K_{\max }$ as the temperature decreases. At these low temperatures the rate of $2 \mathrm{D}$ nucleation is also expected to be rapid on the prism faces, which should then also result in $K_{\text {prism }}=K_{\max }$. With this, Kuroda and Lacmann argue on simple geometrical grounds that diffusion effects are sufficient to produce the observed 
values of $L_{a} / L_{c}$ in this region.

We see from Fig. 3, however, that the KL model is incorrect at low temperatures, and that we must have $K_{\text {prism }}$ $\ll K_{\text {basal }}$ below $T=-25^{\circ} \mathrm{C}$ in order to explain the observations. Assuming that 2D nucleation on the prism faces is not a limiting factor in the growth at these temperatures, the most likely explanation for the observations is that the sticking probability on the prism facets is considerably less than unity, approximately $\alpha_{\text {prism }} \approx 0.05$, while the sticking probability to the basal faces is near $\alpha_{\text {basal }} \approx 1$. We cannot offer a convincing physical argument for why we should have $\alpha_{\text {prism }} \ll \alpha_{\text {basal }}$ below $T=-25^{\circ} \mathrm{C}$, but one intriguing possibility is the "poisoning" of the available lattice sites by the solvent gas molecules. Evidence for this possibility has been suggested previously by Beckmann [32].

As described by Kuroda and Lacmann $[18,19]$ in the KL model, the onset of disordering brings with it a substantial change in the kinetic coefficient, from a large value of $K$ just above the transition temperature to a much smaller value below. This arises because the adhesive growth rate is high on the disordered surface, but can be very small in the absence of the disordered layer. On the basal faces, the measured disordering transition at $T \approx-15^{\circ} \mathrm{C}$ [21] is in fairly good agreement with the KL model, which then explains the transition to a very low basal kinetic coefficient as the temperature is lowered to $T \approx-15^{\circ} \mathrm{C}$. The slight discrepancy in the disordering temperature between the $\mathrm{KL}$ model and the recent $\mathrm{X}$-ray scattering data [21] may possibly be reconciled if the kinetic coefficient depends on solvent gas, since the growth measurements were done in air while the x-ray data were obtained with no solvent gas present above the ice surface [33]. The transition from small $K$ to $K \approx K_{\max }$ on the basal faces below $T \approx-25^{\circ} \mathrm{C}$ then arises, according to the $\mathrm{KL}$ model, from the onset of rapid 2D nucleation at these lower temperatures.

A serious problem with the KL model is apparent when considering the prism faces, however. In order to explain the growth data, the KL model assumes that the disordering transition on the prism faces occurs at a substantially lower temperature than for the basal faces. This assumption is not borne out by the recent $\mathrm{x}$-ray data, in which a disordering transition is found at $T \approx-12{ }^{\circ} \mathrm{C}$ for the prism face, i.e., at a higher temperature than for the basal face. Although various measurements of the disordered layer on ice have yielded conflicting results [34], it seems unlikely from the sum of these data that the disordering transition on the prism face occurs below $T=-15^{\circ} \mathrm{C}$. This issue remains largely unresolved.

In summary, we have described above a relatively simple Green's function approach for modeling cylindrically symmetric crystal growth. This approach can adequately model the hexagonal prism growth of ice crystals, as long as there is not significant hollowing of the prism faces. We used this approach to model existing ice crystal growth data, and produced a measure of the surface kinetic coefficients of the basal and prism faces as a function of temperature, around a single supersaturation level. Clearly, additional growth measurements at different supersaturations and using different solvent gases would be very useful for unraveling the unusual growth behavior and surface structure of ice.
[1] For reviews, see E. Ben-Jacob, Contemp. Phys. 38, 205 (1997); 34, 247 (1993); M. C. Cross and P. C. Hohenberg, Rev. Mod. Phys. 65, 851 (1993).

[2] A. Karma and W.-J. Rappel, Phys. Rev. Lett. 77, 4050 (1996).

[3] Y. Saito, G. Goldbeck-Wood, and H. Muller-Krumbhaar, Phys. Rev. Lett. 58, 1541 (1987); Phys. Rev. A 38, 2148 (1988).

[4] E. Yokoyama and T. Kuroda, Phys. Rev. A 41, 2038 (1990); E. Yokoyama, J. Cryst. Growth 128, 251 (1993).

[5] J. A. Sethian and J. Strain, J. Comput. Phys. 98, 231 (1992).

[6] R. Almgren, J. Comput. Phys. 106, 337 (1993).

[7] T. Ihle and H. Muller-Krumbhaar, Phys. Rev. E 49, 2972 (1994).

[8] A. Karma and W.-J. Rappel, Phys. Rev. E 57, 4323 (1998), and references therein.

[9] N. Provatas, N. Goldenfeld, and J. Dantzig, Phys. Rev. Lett. 80, 3308 (1998).

[10] M. Conti, Phys. Rev. E 56, 3197 (1997).

[11] B. T. Murray, A. A. Wheeler, and M. E. Glicksman, J. Cryst. Growth 154, 386 (1995).

[12] A. Schmidt, J. Comput. Phys. 125, 293 (1996), and references therein.

[13] J. G. Dash, H. Fu, and J. S. Wettlaufer, Rep. Prog. Phys. 58, 115 (1995).

[14] M. B. Baker and J. G. Dash, J. Geophys. Res. 99, 10621 (1994).
[15] Y. Furukawa and H. Nada, in Advances in the Understanding of Crystal Growth Mechanisms, edited by T. Nishinaga et al. (Elsevier, Amsterdam, 1997).

[16] Y. Furukawa and H. Nada, J. Phys. Chem. B 101, 6170 (1997).

[17] C. A. Knight, J. Geophys. Res. 101, 12921 (1996).

[18] Ice crystal growth from vapor is reviewed by T. Kobayashi and T. Kuroda, in Morphology of Crystals, Part B, edited by I. Sunagawa (Terra Scientific, Tokyo, 1987).

[19] T. Kuroda and R. Lacmann, J. Cryst. Growth 56, 189 (1982); T. Kuroda, ibid. 99, 83 (1990).

[20] J. Nelson and C. Knight, J. Atmos. Sci. 55, 1452 (1998).

[21] A. Lied, H. Dosch, and J. H. Bilgram, Phys. Rev. Lett. 72, 3554 (1994); H. Dosch, A. Lied, and J. H. Bilgram, Surf. Sci. 327, 125 (1995)

[22] A. Yamashita, Kisho Kenkyu Noto, Met. Soc. Japan 123, 47 (1974).

[23] Y. Saito, Statistical Physics of Crystal Growth (World Scientific, Singapore, 1996).

[24] J. E. Taylor and J. W. Cahn, Physica D 112, 381 (1998).

[25] M. Adda Bedia and M. Ben Amar, Phys. Rev. E 51, 1268 (1995).

[26] J. S. Langer, Rev. Mod. Phys. 52, 1 (1980).

[27] J. D. Jackson, Classical Electrodynamics, 2nd ed. (Wiley, New York, 1975).

[28] We used QuickField 4.1, developed by the Tera Analysis Company. 
[29] W. B. Burton, N. Cabrera, and F. C. Frank, Philos. Trans. R. Soc. London, Ser. A 243, 299 (1951).

[30] V. W. Keller and J. Hallett, J. Cryst. Growth 60, 91 (1982).

[31] N. J. Bacon et al., J. Geophys. Res. 103, 13763 (1998).
[32] W. Beckmann, J. Cryst. Growth 58, 443 (1982).

[33] H. Dosch (private communication).

[34] M. Elbaum, S. G. Lipson, and J. G. Dash, J. Cryst. Growth 129, 491 (1993), and references therein. 